\title{
Autoimmune pancreatitis as a component of autoimmune polyglandular syndrome
}

\author{
Grażyna Piotrowicz', Beata Stępieńn ${ }^{2}$ Agnieszka Klufczyńska ${ }^{1}$ \\ ${ }^{1}$ Gastroenterology Department, Hospital of Ministry of Home Affairs, Gdansk, Poland \\ 2Department of Internal Medicine and Gastroenterology, Central Clinical Hospital of Ministry of Home Affairs, Warsaw, Poland
}

Gastroenterology Rev 2017; 12 (1): 66-67

DOI: $10.5114 /$ pg.2016.62151

\begin{abstract}
Address for correspondence: Grażyna Piotrowicz MD, PhD, Gastroenterology Department, Hospital of Ministry of Home Affairs, 4/6 Kartuska St, 80-104 Gdansk, Poland, phone: +48 602784 507, e-mail: piotrowicz.grazyna@interia.eu
\end{abstract}

Autoimmune pancreatitis (AIP) is a rarely diagnosed autoimmune illness with a characteristic clinical, histological, and radiological image [1]. The frequency of AIP occurrence is estimated to be $1.9-4 \%$; however, this illness must be differentiated from the pancreatitis [2, 3].

The illness has two histological clinical sub-types: type 1: lymphoplasmacytic sclerosing pancreatitis (LPSP) - it accompanies other autoimmune illnesses: primary sclerosing cholangitis, primary biliary cirrhosis, Sjögren syndrome, inflammatory bowel disease, diabetes type 1 ; type 2 : idiopathic duct-centric pancreatitis (IDCP) - isolated autoimmune pancreatitis [4, 5].

Recent reports indicate that patients with diagnosed autoimmune pancreatitis are more prone to develop ulcerative colitis (CU) as was suggested by Korean scientists [6]. The diagnosis can be based on the following diagnostic criteria for autoimmune pancreatitis [7]. It has been postulated in recent years that autoimmune pancreatitis belongs to the systemic disease group related to IgG4, otherwise called the IgG4-associated systemic disease (ISD)/lgG4-related sclerosing disease [8]. Extrapancreatic lesions occur in $80 \%$ of AIP patients and include sclerosing cholangitis (58\%), lymphadenopathy recess lung (28\%), inflammation of the salivary glands and tear ducts (15-39\%), interstitial pneumonia (8-13\%), retroperitoneal fibrosis, and tubulointerstitial nephritis. Elevated levels of IgG4 in plasma cells have also been reported in pseudoinflammatory liver, breast, mediastinum, orbit tumours, and in the pituitary gland and prostate inflammation $[9,10]$.

Due to the fact that autoimmune pancreatitis type 1 (LPSP) may be accompanied by other autoimmune diseases, AIP may be one of the autoimmune polyendocrinopathies, autoimmune polyglandular syndrome (APS) type $1,2,3$, and 4 [11].

In every person suffering from autoimmune disease, particularly of the endocrine glands, the possibility of
APS occurrence needs to be considered in the differential diagnosis, testing for the possible coexistence of diabetes type 1, thyroid dysfunction, Addison's disease, celiac disease, and autoimmune hepatitis, also smooth muscle, and antigens LMC- 1 and SLA is recommended [12, 13].

The patient, aged 72 years, was first interviewed in 2006 because of obstructive jaundice, which occurred in the course of the pancreatic head tumour diagnosis based on magnetic resonance cholangiopancreatography (MRCP) and endoscopic retrograde cholangiopancreatography (ERCP) examination (April 2006) (Figure 1). On the basis of the clinical image and imaging, the patient was qualified for surgery, and the intraoperative excerpt from the head of the pancreas exhibited inflammatory and neoplastic lesions. The microscopic image was consulted again with pathomorphologists; there was no description of any atypical characteristics or mitotic activity (Ki 67-), and carcass tissues revealed the presence of lymphoid cells, plasma, and eosinophils. Infiltration of eosinophils was also found in the wall of the removed gallbladder.

In addition, the patient had been treated with vitamin $B_{12}$ since 1995 due to Addison-Biermer disease, and since 1988 also Graves Basedow disease with infiltrative ophthalmopathy treated with Solu Medrolu pulses in the Department of Endocrinology in 2001 and 2003. The patient also underwent positron emission tomography examination (July 2006), which revealed no outbreaks of increased metabolic activity. In 2007 the patient was hospitalised in the Department of Internal Medicine because of weakness, loss of appetite, pain in the intra-abdominal region, and weight loss. The imaging examinations revealed: in the ultrasound examination of the abdominal cavity - the expansion of common bile duct (CBD) in the parahilar region up to $14 \mathrm{~mm}$, and a lesion in the head of the pancreas $37 \times 25 \mathrm{~mm}$, confirmed by magnet- 


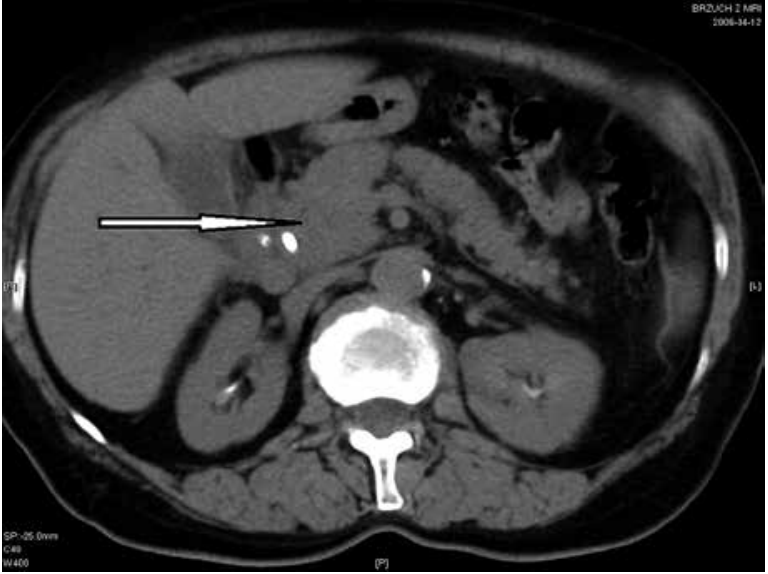

Figure 1. Magnetic resonance imaging - 2006

ic resonance imaging (MRI) with MRCP option. The fine needle aspiration biopsy examination revealed infiltrated inflamed tissue strips. Laboratory studies of peripheral blood revealed eosinophilia $22 \%$, as in previous studies of peripheral blood. Due to the overall clinical picture it was decided to assay lgG4, the value of which was six times higher than normal.

Steroids were introduced for a period of 6 months (Encorton), which resulted in general improvement and regression of inflammatory changes in the MRI examination (2007, 2009, and 2012) (Figure 2).

During further examinations, the level of IgG4 remained within the upper limit of the norm.

The patient remains under endocrinological and endoscopic supervision due to the disappearance of the gastric mucosa in the course of the A-B disease.

In 2010 gastrofiberoscopy with collected specimen revealed a neuroendocrine tumour (GEP-NET) meshwork with enetero-chromophilic cells with low mitotic activity in endometrial biopsies of stomach. Chromogranin was determined to be more than three times the accepted laboratory standard. A recent gastrofiberoscopy with biopsy of the gastric antral mucosa and the prepyloric region revealed the proliferation of enetero-chromophilic cells with increased production of chromogranin, alongside the deterioration of gastric mucosa.

This case is an example of type 1 AIP (LPSP), where pancreatic symptoms occurred more than 10 years after the first symptoms of APS, which should sensitise us to this type of patient and, perhaps, in the diagnosis of pancreatic patients in this category, increase our oncological vigilance and make us pay more attention to AIP diagnostics in accordance with the general criteria in the first place.

\section{Conflict of interest}

The authors declare no conflict of interest.

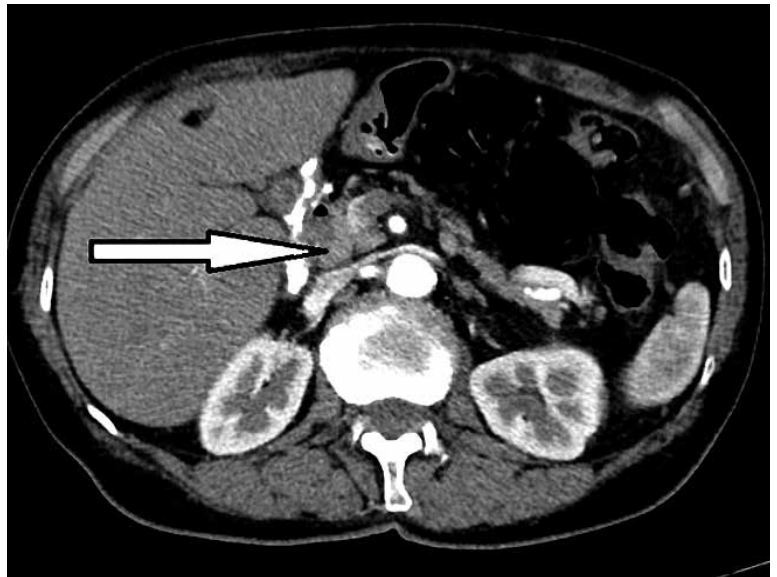

Figure 2. Magnetic resonance imaging - 2012

\section{References}

1. Ratyński T, Degowska M, Milewski J, Rydzewska G. Autoimmune pancreatitis - case report, diagnostic, treatment. Przegl Gastroenterol 2006; 1: 49-53.

2. Kamisawa T, Takuma K, Egawa N, et al. Autoimmune pancreatitis and IgG4-related sclerosing disease. Nat Rev Gastroenterol Hepatol 2010; 7: 401-9.

3. Cirocchi R, Santoro A, Corsi A, et al. Autoimmune pancreatitis: a case of difficult diagnosis. Przegl Gastroenterol 2015; 10: 51-3.

4. Chari ST, Kloeppel G, Zhang L, et al. Histopathologic and clinical subtypes of autoimmune pancreatitis: the Honolulu Consensus Document. Pancreatology 2011; 10: 664-72.

5. Deshpande V, Gupta R, Sainani N, et al. Subclassification of autoimmune pancreatitis: a histologic classification with clinical significance. Am J Surg Pathol 2011; 35: 26-35.

6. Park SH, Kim D, Ye BD, et al. The characteristics of ulcerative colitis associated with autoimmune pancreatitis. J Clin Gastroenterol 2013; 47: 520-5.

7. Naitoh I, Nakazawa T, Ohara H, et al. Comparative evaluation of the Japanese diagnostic criteria for autoimmune pancreatitis. Pancreas 2010; 39: 1173-9.

8. Kamisawa T, Takuma K, Egawa N, et al. Autoimmune pancreatitis and IgG4-related sclerosing disease. Nat Rev Gastroenterol Hepatol 2010; 7: 401-9.

9. Pezzilli R, Pagazano N. Pathophysiology of autoimmune pancreatitis. World J Gastrointest Pathophysiol 2014; 5: 11-7.

10. Milosavljevic T, Kostic-Milosavljevic M, Jovanovic I, et al. Extraintestinal manifestations of autoimmune pancreatitis. Dig Dis 2012; 30: 220-3.

11. Hansen MP, Kahaly GJ. Autoimmune polyglandular syndromes. Dtsch Med Wochenschr 2013; 138: 319-30.

12. Okazaki K, Uchida K, Miyoshi H, et al. Recent concepts of autoimmune pancreatitis and IgG4-related disease. Clin Rev Allergy Immunol 2011; 41: 126-38.

13. Pasierbek-Kohutek A, Gutkowski K, Barczyk A, et al. Autoimmune pancreatitis as a systemic, protracted and potentially fatal disease. Przegl Gastroenterol 2013; 8: 71-6.

Received: 6.07.2015

Accepted: 2.03 .2016 